\title{
Innovación Pedagógica y Elaboración de una Guía de Aprendizaje en Tecnología Farmacéutica Industrial
}

\author{
$M^{a}$ A. Peña, Guillermo Torrado, Pilar Bustamante y Begoña Escalera \\ Universidad de Alcalá de Henares, Facultad de Farmacia, Departamento de Farmacia y \\ Tecnología Farmacéutica, 28871 Alcalá de Henares-Madrid (e-mail: angeles.pena@uah.es)
}

\begin{abstract}
Resumen
El objetivo del presente trabajo, es diseñar un plan didáctico siguiendo estrategias de enseñanzaaprendizaje marcadas por las orientaciones europeas y plantear un sistema de evaluación que responde a las exigencias del modelo de formación implantado. Se incluye en el estudio aspectos relacionados con la formación por competencias. La aplicación de esta guía consigue incluir mejoras adaptadas a las necesidades detectadas a partir de sucesivos cursos académicos y que pueden seguir usándose hasta que se pongan en marcha los nuevos planes de estudio. Además, en el ámbito del alumnado se obtuvo un gran interés por la asignatura con un alto grado de motivación y compromiso. Muchas de estas cuestiones sirven como punto de partida y de reflexión previa, e incluso ayudan a mejorar el plan docente de la asignatura.
\end{abstract}

Palabras clave: guía de aprendizaje, competencias, evaluación, plan didáctico, innovación

\section{Pedagogical Innovation and Elaboration of a Learning Guide In Industrial Pharmaceutical Technology}

\begin{abstract}
The objective of this work is to design a didactic plan following strategies of education-learning marked by the European convergence process and to propose an evaluation system that responds to the exigencies of the model implanted of formation. In addition to that, aspects related to the formation by competitions are discussed. The application of this guide is able to include improvements adapted to the necessities detected from successive academic courses and that can be used until the new curriculum is implemented. Additionally, great interest for the course was observed among students, with a high degree of motivation and commitment. Many of these questions serve as the departure point and as previous reflection, and even help in improving the educational plan of the course.
\end{abstract}

Keywords: learning guide, competences, evaluation, didactic plan, innovation 


\section{INTRODUCCIÓN}

El presente trabajo recoge el diseño y elaboración de un plan didáctico o programa de aprendizaje de la asignatura Tecnología Farmacéutica Industrial (3 créditos teóricos y 1,5 prácticos), presente en los actuales planes de estudio de la Licenciatura de Farmacia de la Universidad de Alcalá (Madrid, España). Se trata de un proceso complejo y coordinado con otras materias, para conseguir el aprendizaje de calidad del alumno (Gimeno, 2005). La guía de aprendizaje es un documento normalizado que contiene la información necesaria para el desarrollo completo e integral de una asignatura y sigue la siguiente estructura:

a. Identificación de la asignatura (descripción)

b. Presentación o justificación (contextualización)

c. Propósitos/Objetivos/Competencias

d. Contenidos

e. Metodología de enseñanza-aprendizaje. Actividades formativas

f. Evaluación

g. Bibliografía y recursos

Esta asignatura optativa se enmarca en la titulación de la Licenciatura de Farmacia de la Universidad de Alcalá:

- Ubicación en el plan de estudios. Se imparte durante los últimos cursos académicos del segundo cuatrimestre de la licenciatura. Dicha ubicación obedece a la necesidad de la adquisición previa, por parte de los alumnos, de una serie de conocimientos, habilidades y destrezas en cursos anteriores sobre: principios activos, excipientes farmacéuticos, maquinaria industrial, procesos de elaboración de medicamentos, farmacotécnica de las formas farmacéuticas a nivel industrial y magistral, control de calidad de las formas farmacéuticas a través de ensayos farmacotécnicos y biológicos, incluyendo los exigidos por la RFE.

- Interrelación con otras asignaturas, especialmente con, Principios de Farmacia Galénica, Farmacia Galénica, Biofarmacia, Formulación de Medicamentos, Farmacia Clínica y Gestión y Planificación Farmacéutica y Control de Calidad de Medicamentos.

- Aportes a su perfil de egreso. Desarrollo integral del alumno encaminado a su futuro profesional en la industria farmacéutica mediante la adquisición de los fundamentos tecnológicos necesarios para una correcta fabricación industrial.

Con respecto a los propósitos de la asignatura, ésta se dirige específicamente a la formación para adquirir conocimientos, técnicas y habilidades en la fabricación de medicamentos a nivel industrial y obtener una primera aproximación de cómo es un laboratorio farmacéutico. Otros objetivos se centran en ofrecer una panorámica actual del mundo de la industria farmacéutica (Carbonell, 2003).

El proceso de auto-aprendizaje, el trabajo continuo y organizado, convergerá en la adquisición de una mayor competitividad y mejor calidad en la formación de los alumnos (Margalef y Torne, 2007). Con una asimilación de conceptos, destrezas y estrategias correctas de estas prácticas se conseguirá un aprendizaje eficaz y se dotará a los alumnos de un aprendizaje de mayor calidad. A continuación se describen las competencias, entendidas como combinación de conocimientos, habilidades, procedimientos, estrategias, actitudes y valores. Según Perrenoud (2004) competencia es la capacidad de movilizar varios recursos cognitivos para hacer frente a un tipo de situación. Se dividen en: genéricas, transversales y específicas.

\section{Genéricas}

Desarrollar capacidad para el análisis y la síntesis

Desarrollar habilidades tecnológicas (herramientas informáticas)

Desarrollar un pensamiento crítico y reflexivo sobre el aprendizaje. Capacidad para la autocrítica Propiciar el trabajo en equipo y el respeto hacia los demás.

Favorecer la iniciativa y la capacidad emprendedora

Motivación de logro 


\section{Transversales}

Asimilar los conceptos y planteamientos que se expresan en el programa

Integrar los conocimientos en nuestro pensamiento

Establecer una interrelación con asignaturas comunes a fin de dirigir la formación de estos estudiantes en la industria farmacéutica, sobre todo con Control de Calidad en la Industria Farmacéutica

\section{Específicas}

Resolver situaciones o conflictos en el laboratorio farmacéutico

Conocer la evolución del proceso en el desarrollo de una forma farmacéutica

Dominar técnicas e instrumentos de fabricación de medicamentos

Adoptar una actitud reflexiva frente a los resultados experimentales obtenidos

La planificación de la enseñanza basada en competencias debe tener en cuenta el entorno social, laboral, económico...y según todos estos determinantes curriculares planificar teniendo en cuenta lo que indica el Real Decreto 1393/2007.

Por otra parte, la asignatura está dividida en un programa teórico (12 temas) y un programa práctico (3 temas). Se indican los temas de cada parte:

\section{PROGRAMA TEÓRICO:}

TEMA 1. Origen, desarrollo y objetivos de la producción industrial de medicamentos. La investigación y el desarrollo galénicos. La planta piloto y el cambio de escala.

TEMA 2. Intervención de los estados en la autorización y producción de medicamentos. La agencia del medicamento. El registro de especialidades farmacéuticas. Aspectos básicos sobre la calidad en la industria farmacéutica.

TEMA 3. El laboratorio farmacéutico: Aspectos generales. Factores que influyen en su ubicación. Edificios y zonas. Instalaciones, equipos y personal.

TEMA 4. Presión. Aire comprimido: Características, unidades de medida y aplicaciones en un laboratorio farmacéutico. Compresores de aire: aspectos teóricos. Instalaciones industriales. Equipos de medida.

TEMA 5. Vacío: Aspectos teóricos y aplicaciones en un laboratorio farmacéutico. Bombas de vacío: Clasificación y tipos. Equipos de medida.

TEMA 6. Refrigeración: Métodos de producción de frío. Calefacción: Transmisión del calor. El vapor de agua y otros agentes de calefacción.

TEMA 7. Climatización del aire. Estado higrométrico. Diagrama psicrométrico. Deshumidificación y humidificación del aire. Áreas especiales.

TEMA 8. Esterilización del aire. Salas estériles. Flujo turbulento y flujo laminar. Mantenimiento y control de la esterilidad.

TEMA 9. Tratamiento del agua. Instalaciones industriales para la obtención de agua purificada y agua para inyección. Validación de procesos de producción de agua para uso farmacéutico.

TEMA 10. Diseño de instalaciones y equipos para la fabricación industrial de medicamentos sólidos por vía oral.

TEMA 11. Diseño de instalaciones y equipos para la producción industrial de formas líquidas orales

TEMA 12.- Envasado

TEMA 13.- Bibliografía

PROGRAMA PRÁCTICO:

TEMA 1. Procedimiento normalizado de trabajo de una máquina de comprimir.

TEMA 2. Validación de un proceso de compresión

TEMA 3. Protocolo de fabricación de medicamentos orales sólidos (comprimidos) y líquidos (solución).

\section{METODOLOGÍA}

En la guía de aprendizaje se describe en el apartado e la metodología de enseñanza-aprendizaje (actividades formativas), los métodos llevados a cabo se han seleccionado para poner en marcha el principal objetivo de esta innovación docente que es conseguir un aprendizaje coloborativo y 
reflexivo de los estudiantes. Se ha considerado la siguiente organización y usos de espacios de aprendizaje (Imbernon y Medina, 2006):

X El número de créditos teóricos de la asignatura es de 3, que se dividen en:

- 2,2 créditos de clases presenciales (clases magistrales)

- 0,8 créditos de clases no-presenciales (realización y exposición de trabajos en grupo)

* El número de créditos prácticos de la asignatura es de 1,5. Las prácticas tienen lugar en el laboratorio. Previamente a su realización, de forma individual, el profesor imparte un seminario de 1 hora de duración, en el que se explica el protocolo de trabajo a seguir. El protocolo se entrega por escrito al alumno (cuaderno de prácticas), junto con la información detallada para realizar las prácticas. En el cuaderno aparecen una serie de cuestiones que deben ser resueltas y corregidas por los profesores (actividad no-presencial).

\section{Clases teóricas presenciales:}

El profesor expone en forma de clase magistral los 12 temas que componen la asignatura en 22 sesiones. Para un perfecto desarrollo de la asignatura, el docente tiene que tener en cuenta una serie de factores como son: conocer a la audiencia, conocer el nivel del alumnado, correcta utilización de imágenes y soportes gráficos audiovisuales, el tiempo de cada clase, los estilos de enseñanza...Con el fin de conseguir un aprendizaje activo (Barkley et al., 2007) se recurre a actividades de resumen, síntesis y evaluación, que permiten al grupo reforzar todos los aprendizajes realizados durante la sesión. Además, a fin de romper la fatiga del aula, se incluyen estrategias participativas, como puzzle de grupos, red de conceptos, técnica de colocar estructuras...Debemos favorecer el aprendizaje reflexivo-experiencial (Kolb, 1984). Al comienzo del cuatrimestre, se harán los grupos con cuatro a seis personas, de forma voluntaria, para que comiencen a realizar sus trabajos; en las tutorías grupales (asistencia obligatoria de todos los alumnos) se discutirá la marcha de los trabajos, se aclararán dudas y se expondrán los trabajos realizados al final del cuatrimestre.

Clases teóricas no-presenciales:

Los alumnos elaborarán trabajos en grupo. En este trabajo deben recopilar bibliografía, consultar la web, puesta en común y discusión con el grupo de los materiales recogidos y por último redacción y exposición de los trabajos. En este tiempo los alumnos también deben preparar la evaluación. Para ello cuentan con la bibliografía facilitada (escrita/on-line) por los profesores, además del material complementario o de apoyo elaborado por ellos y que estaría a disposición de los alumnos en un libro o dossier editado por los profesores que imparten la materia, que incluye un resumen más detallado de lo explicado en clase con la bibliografía completa. El libro se ha incluido en el presente curso escolar a fin de contribuir a un aprendizaje más activo y autónomo. Además, los alumnos cuentan con tutorías on-line y tutorías personalizadas (individuales) en éstas, se puede acudir al despacho del profesor para consultar o aclarar aspectos relacionados con el desarrollo de la asignatura en cualquiera de sus vertientes.

El diseño, elaboración y exposición de los trabajos se realizará en grupos de 4 a 6 alumnos. La elaboración del trabajo corresponderá con los contenidos desarrollados en los temas 10 y 11 del programa teórico de la asignatura, apoyándose en los conocimientos de los temas anteriores. Durante la fase de elaboración los alumnos deberán acudir a los profesores de la asignatura para comprobar la marcha del trabajo (tutorías on-line y tutorías personalizadas), y si fuera necesario, efectuar las correcciones oportunas. Es imprescindible la revisión anticipada del trabajo para poder exponerlo posteriormente en el aula al resto de compañeros (Onrubia, 2008).

Los alumnos deberán entregar el trabajo por escrito antes de su exposición oral. La exposición debe comportar el uso de material audiovisual apropiado, lo que a su vez conlleva la elaboración de un póster (presentación estática) o unas transparencias o diapositivas (presentación dinámica). Se valorará positivamente la creatividad y las presentaciones atractivas y bien desarrollas (con el uso de herramientas como el PowerPoint).

El trabajo presentará un número mínimo de 6 hojas y máximo de 10. A continuación se indican los contenidos en los que se puede trabajar: 


\section{PROGRAMA TRABAJO EN GRUPO:}

Formas farmacéuticas orales sólidas: comprimidos

Formas farmacéuticas orales sólidas: comprimidos efervescentes

Formas farmacéuticas orales líquidas: de pequeño volumen

Formas farmacéuticas orales líquidas: de gran volumen

Formas farmacéuticas orales semisólidas: pomadas

Formas farmacéuticas rectales: supositorios

Formas farmacéuticas orales líquidas estériles: de pequeño volumen y gran volumen

Formas farmacéuticas de uso veterinario

Deberá tener la siguiente estructura:

o Título del trabajo

- Nombre de los participantes

o Curso académico

o Asignatura

o Introducción y descripción del tema

o Objetivos

o Métodos

o Discusión

- Bibliografía

o Anexos (Tablas y Figuras)

Finalmente, con respecto a la evaluación, se deben indicar previamente a la misma los criterios de evaluación ya que son fundamentales para orientar a profesores y estudiantes en el seguimiento y desarrollo de logros y competencias. Evitan la arbitrariedad y permiten un proceso de autoevaluación. En la evaluación se tiene en cuenta la teoría y las prácticas, según:

\section{TEORÍA}

La evaluación consiste en la superación de un examen al final del cuatrimestre, valorado en ocho puntos. Este examen se divide en dos partes, un test de 40 preguntas, con una puntuación máxima de 6 puntos, para conseguir la mitad de esa puntuación se debe superar el 70\%, y dos preguntas a desarrollar con una valoración de 2 puntos.

El trabajo suma los dos puntos finales. Se valora el trabajo escrito con un $75 \%$ del peso total $(1,5$ puntos) y la presentación oral (0,5 puntos).

\section{PRÁCTICAS}

Los alumnos deben realizar obligatoriamente las prácticas (1,5 créditos) para aprobar la asignatura. Posteriormente, se valoran las cuestiones incluidas en el cuaderno de prácticas de laboratorio. Si se han superado ambos requisitos, la evaluación de las mismas se realiza contestando a las preguntas del test del examen final, que se corresponden con las prácticas. Entre las 40 preguntas, 10 pertenecen a las prácticas. Se deben tener muy en cuenta los objetivos y competencias señaladas, para que la evaluación sea de conocimientos, habilidades, destrezas y actitudes.

\section{RESULTADOS Y DISCUSÍON}

Se hicieron unas preguntas a los estudiantes al inicio de poner en marcha esta guía docente para conocer su nivel de implicación en el proceso de innovación pedagógica, sus conocimientos previos y lo que esperan de él. Los resultados obtenidos muestran inicialmente un claro escepticismo sobre los cambios introducidos con respecto a la forma tradicional de impartir una materia. Aunque su nivel de confianza era alto y pensaban que las destrezas conseguidas les serian útiles para desarrollar su futuro profesional en la industria farmacéutica. 
Al finalizar el curso, muchos estudiantes se mostraron totalmente comprometidos con el proceso y la asignatura. Su nivel de dedicación y el tiempo dedicado al estudio así como a la realización de trabajos en grupo fue inesperadamente alto. Esto se vio reflejado en los resultados de la evaluación, por un lado las calificaciones y por otro la asistencia a tutorías y la correcta elaboración y puesta en escena de los trabajos grupales. Con respecto a los trabajos presentados todos tuvieron una calidad alta lo cual fue gratamente recibido por los profesores, ya que se considera como una respuesta positiva de los estudiantes por parte de lo que se le había demandado.

Por tanto, los resultados han sido muy satisfactorios, el porcentaje de suspensos ha disminuido. Solamente los estudiantes pertenecientes al programa de intercambio ERASMUS (alumnos de lengua no castellana), mostraron resultados académicos parecidos o incluso peores que en años anteriores, su nivel de implicación fue nulo, no hubo ninguna implicación con las actividades propuestas.

\section{CONCLUSIÓN}

Se ha desarrollado una guía de aprendizaje para implementar la asignatura de Farmacia y Tecnología Farmacéutica en el curso académico 2007/2008 ( $2^{\circ}$ cuatrimestre). Se piensa que tras la aplicación de esta guía, se podrán incluir mejoras que se adapten a las necesidades detectadas a partir de sucesivos cursos académicos y hasta que se pongan en marcha los nuevos planes de estudio. Las conclusiones son en general de gran interés por la asignatura (es optativa) con un alto grado de motivación y compromiso. Muchas de estas cuestiones sirven como punto de partida y de reflexión previa e incluso para mejorar el plan docente de la asignatura.

La percepción inicial que se ha tenido ha sido de escepticismo, sobre todo debido a una escasa información y poca experiencia previa de los alumnos. Su interés y motivación sin embargo es muy alto en general, lo que compensará esa desconfianza inicial y el esfuerzo personadle los docentes.

\section{REFERENCIAS}

Barkley , E., K.P. Cross y C. Howell; Técnicas de aprendizaje colaborativo. $1^{\mathrm{a}}$ Ed. Ministerio de Educación y Ciencia. Morata. Madrid. España (2007).

Carbonell, J.; La aventura de innovar. El cambio en la escuela. Rev. Ped.: 34(71), 469-473 (2003).

Gimeno, J.; La educación que aún es posible. $1^{\mathrm{a}}$ Ed. Madrid (Morata) (2005)

Imbernon, F. y J.L. Medina; Metodologías participativas en la universidad. Documento de trabajo. Programa de formación del profesorado universitario (2006)

Kolb, D.A.; Experiential learning: Experience as the source of learning and development. $1^{\mathrm{a}}$ Ed. Prentice-Hall Inc. New Jersey (1984).

Margalef, L. y E. Torne; Estrategias de innovación docente para favorecer el aprendizaje autónomo de los estudiantes de la Universidad de Alcalá. Obras Colectivas, Educación 01.UAH. España (2007).

Onrubia, J.; Aprender y enseñar en entornos virtuales: actividad conjunta, ayuda pedagógica y construcción del conocimiento. RED http://www.um.es/ead/red/M2/ (2008)

Perrenoud , P.; Diez nuevas competencias para enseñar. $1^{\mathrm{a}}$ Ed. Grao. Barcelona. España (2004) 